\title{
STEM Majors' Perceptions of Racism and Sexism in STEM
}

\author{
Katherine Rainey ${ }^{1}$, Melissa H. Dancy ${ }^{1}$, Roslyn Mickelson ${ }^{2}$, Elizabeth Stearns ${ }^{2}$, and Stephanie Moller ${ }^{2}$ \\ ${ }^{1}$ Department of Physics, University of Colorado, Boulder, CO 80309 \\ ${ }^{2}$ Department of Sociology, University of North Carolina - Charlotte, Charlotte, NC 28223
}

\begin{abstract}
We present a small part of a larger interview study of approximately 150 college seniors with diverse gender and race backgrounds completing a STEM degree. In the interviews, students were asked if the experience of being a STEM major was different for people of different genders and races. We report students' perceptions of race and gender differences and their explanations for these differences. Interviewees reported differences based in individuals (i.e. women are not as interested in STEM) as well differences based in sexism and racism (i.e. white men are assumed to be more competent). There were also many interviewees who did not recognize any differences based on gender or race. We found that white men were the least likely to recognize differences for people of other genders and races, whereas women of color were the most likely to recognize these differences. Notably, interviewees were more likely to recognize gender differences than racial ones.
\end{abstract}

Keywords: Gender, Race, Sexism, Racism, Representation

\section{INTRODUCTION}

Women and people of color have been historically excluded from many STEM fields. Though representation has improved [1], women and underrepresented minorities (URMs) still remain largely underrepresented in STEM. Little research has considered student perceptions of the experiences of underrepresented groups in STEM.

Multiple studies have shown that women and people of color have different experiences pursuing STEM fields compared to men and white people. For example, they often experience diminished sense of belonging due to low representation [2] and encounter stereotypes and discrimination [3] as well as microaggressions [4]. These experiences impact persistence and performance [5]. Though studies about race in STEM exist, many more studies exist about differences between men and women in STEM; studies have found that women are evaluated lower than men [6], encounter a culture of masculinity that can push them out of the field [7], and encounter STEM courses that don't align with their preferred learning styles [8].

Though these experiences are well documented, little work has been done to investigate students' perceptions of the existence of these different challenges. Though not related to STEM, the Pew Research Center has conducted national polls that show men and white people in the US are more likely to agree that gender and racial equity has been achieved $[9,10]$.
In addition to a pattern of evidence that privileged groups fail to recognize their own privilege, there are also indications that, despite evidence, privileged groups may believe they themselves are the disadvantaged group [11].

In this paper, we present findings from an interview study regarding student perceptions of gender and racial differences in STEM. We first discuss the interviews conducted. We then present the three common responses to the interview questions, going in depth into responses about perceived racism and sexism mentioned in the interviews. We conclude with recommendations to improve the current approach to improving representation of women and people of color in STEM.

\section{INTERVIEW DATA}

The data presented here was collected as part of a larger study, The Roots of STEM Success Project (https://clas-pages.uncc.edu/rootsofstem/). Interviews were conducted with college seniors who were either majoring in STEM (majors, $\mathrm{N}=142$ ) or who reported starting a STEM major but completed a major in another non-STEM field (leavers, N=59). Students were from public universities in North Carolina (NC), including large research universities, smaller regional schools, and several historically black colleges. To align with the purpose of the study, we oversampled for underrepresented students (in gender and race) in our interview selection process. 
All questions in the interview protocol were designed to elicit student experiences with STEM that led to their decision to major in or leave STEM. In this paper, we focus on two interview questions:

1. Do you think the experience of being a \{STEM major\} is different for men and women?

2. Do you think the experience of being a \{STEM major\} is different for people of different races?

Interviews were recorded and transcribed, then coded using NVivo. The coding rubric was developed through an iterative process involving multiple team members using emergent coding. Due to inconsistencies in interviews, not every person interviewed was asked both questions, or those who were asked both may not have had codable responses. Because of this, the number of respondents for each analysis is not the same. Table 1 presents the number of responses for the following analyses. All statistical tests were done using a test of proportions to calculate a z-score. The z-score conveys the probability that respondents' responses to interview questions are statistically independent of their various demographic characteristics.

TABLE 1. Number of responses for each presented analysis, by demographic group.

\begin{tabular}{c|lcc}
\multicolumn{1}{c}{} & \multicolumn{1}{c}{$\begin{array}{c}\text { White } \\
\text { Students }\end{array}$} & $\begin{array}{c}\text { Students } \\
\text { of Color }\end{array}$ \\
\cline { 2 - 4 } Gender Analysis & Men & 37 & 34 \\
Respondents & Women & 62 & 75 \\
\hline Race Analysis & Men & 27 & 38 \\
Respondents & Women & 62 & 77
\end{tabular}

\section{RESULTS}

Responses fell into three main categories: doesn't notice differences, (2) notes differences but only attributes them to ascribed characteristics of people, and (3) notes sexism/racism impacts. Figure 1 presents the frequency of these responses for both the race and gender analyses. In Sections B-D, we report the most frequently cited explanations given in the interviews.

\section{A. Doesn't Notice Differences}

As Figure 1 shows, many students did not notice gender differences in STEM students' experiences; even more students did not notice racial differences. These responses typically came in the form of outright denial of differences, along with some responses indicating students were unsure. Often times, denial of differences was attributed to the notion that intelligence and ability determine students' experiences in STEM. Referring to

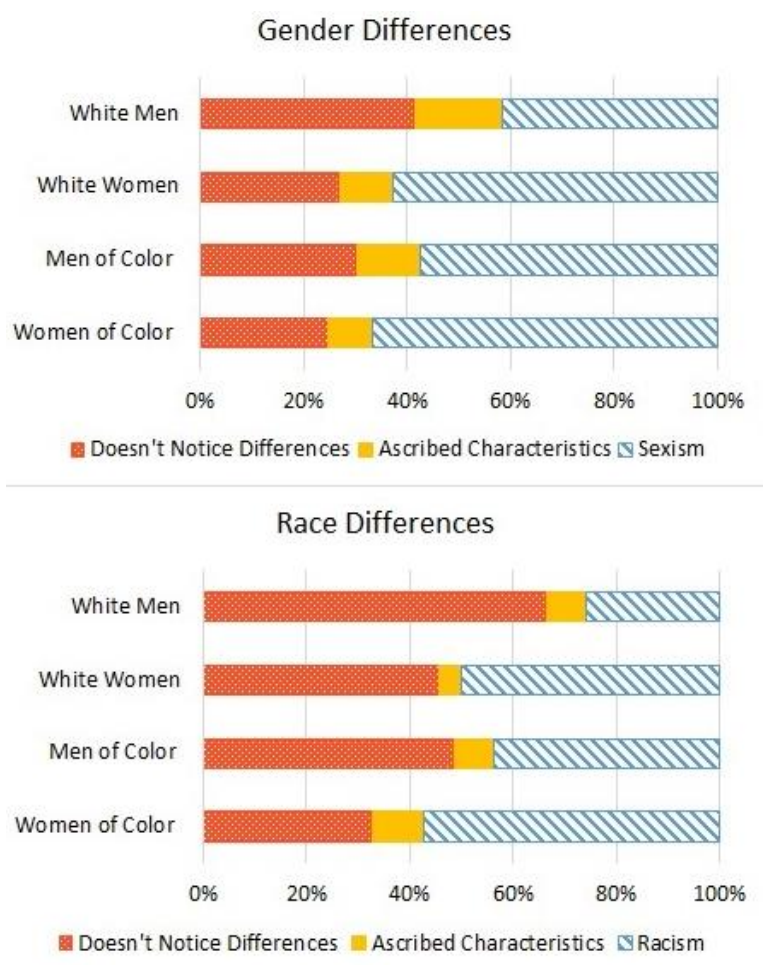

FIG 1. Response rate of explanations for gender and racial differences in STEM.

his computer science major, one black male major said, "most of the things are so technical that is more about what you know than your gender." Referring to race, one Asian male leaver said: "I think for that, it's you know, the skill sets that you have and what you can do and things like that so I don't think the race would matter for that."

Many people used the word "equal" to describe the experiences of people of different genders and races in STEM. A minority of students, most often male or white, felt unable to comment based on their experience as a white or male student. Some students coded in this category said that there was no difference in their own STEM field, but noted it could be different in other STEM fields.

White men (46\%) were the most likely to not notice gender differences compared to other groups $(\mathrm{p}<0.05)$. Further, white men (49\%) were the least likely to notice race differences compared to other groups $(\mathrm{p}<0.05)$.

Comparisons between gender and race analyses show that students are much more likely to not notice race differences than gender differences $(\mathrm{p}<0.001)$.

\section{B. Ascribed Characteristics Account for Race and Gender Differences}

Some students indicated that there were differences in the experiences of STEM students based on race and/or gender, but attributed those differences to 
ascribed characteristics of women or people of color rather than impacts due to the structure or culture of STEM (i.e. sexism/racism).

Women and Some People of Color are not as interested in STEM. A commonly suggested ascribed characteristic cited was that women and some people of color, such as black and Hispanic students, are inherently less interested in science than men, or white and Asian students. For example, one black male major explained, "I think females pay more attention to stuff like history, journalism, and psychology... Guys like math, science, or business." Notably, no women said that women were less interested in science than men are. Most responses regarding race coded this way referred to Asian students being more likely to have an interest in STEM than white students and other students of color.

Women and Some People of Color Work Harder Than Men and People of Other Races. Effort expenditure was mentioned often when discussing gender. Many said that women are naturally harder workers than men but did not attribute this tendency to work harder to any environmental contexts (see below for data combining work ethic with structures). Effort expenditure was also mentioned for race, but at a lower frequency than was mentioned for gender. Comments regarding race and hard work were often focused on people's work ethic and dedication, which several said many URMs often lack.

Biology Impacts Gender Differences. Some students attributed differences to biology; this included differences in the way men and women think as well as physical abilities that may be beneficial for the major (such as physical strength in physical therapy majors).

Different Races Value Education at Different Levels. Though not frequently cited, the value of education was mentioned. This often involved students of color holding weaker education values or prioritizing their family over schooling.

\section{Racism/Sexism Accounts for Race and Gender Differences}

Respondents provided several explanations that attributed differences to sexism or racism. These explanations of differences directly relate to the structure of STEM (i.e. environment, culture, values, norms, etc.) as it stands and were not attributed to any ascribed characteristics.

Being One of the Few Impacts Women and Students of Color. Underrepresentation of women and students of color was mentioned often, as shown in Figure 2, especially by women. Many students stated explicit consequences of that underrepresentation. One

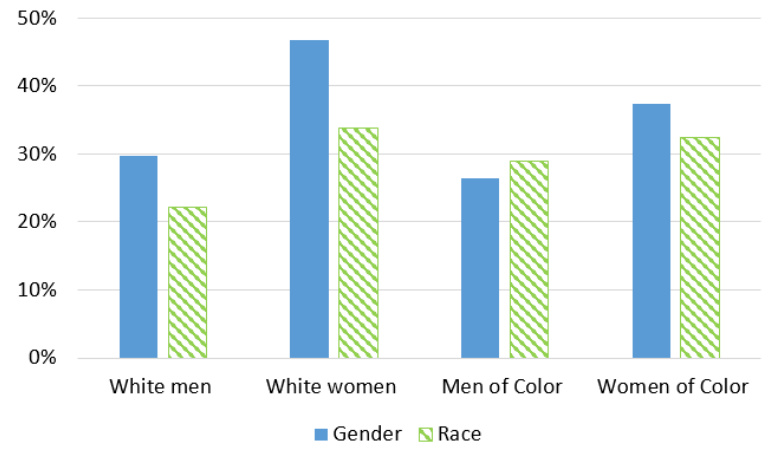

FIG 2. Percent of respondents attributing gender and race differences to low representation of women and people of color.

commonly mentioned factor for both race and gender was intimidation due to being one of the few people of their demographic group in the major. When discussing the number of women in her major, one black woman said, "It's challenging and sometimes discouraging I would say... I don't answer many questions... Women mostly don't speak up in class... We're the quietest ones." She further described how her mixed identity as a black woman impacted her own experience; she felt discouraged due to her underrepresentation and went on to generalize that to the experiences of other women.

Several respondents said underrepresentation created a pressure or motivation for students from underrepresented groups to work harder. This was often due to a fear of confirming negative stereotypes about their demographic group. For example, one white female major described a professor in her department whose views of women motivated her to work harder: "There's this one professor here... his mentality is that women shouldn't be in science. So we kind of have to work against that. I think that makes me work harder because I'm trying to prove to him [that] I can be in science."

Additionally, many said that women and students of color likely lack belonging or feel out of place due to their being surrounded by so many people demographically unlike them. One white female major speculated on what it might feel like to be in the racial minority: "Seeing yourself as an outsider, as a racial minority, I think it would shake your confidence a little or just make you feel like you belong less." Many students mentioned that the lack of role models in STEM, such as female faculty and faculty of color, could be discouraging for those underrepresented in STEM and additionally contribute to lack of belonging for women and people of color.

Men and Some Races Benefit From Societal Beliefs. Societal beliefs about women and men in STEM was the most commonly cited gender difference in our analysis (34\%); $15 \%$ of respondents mentioned societal beliefs 


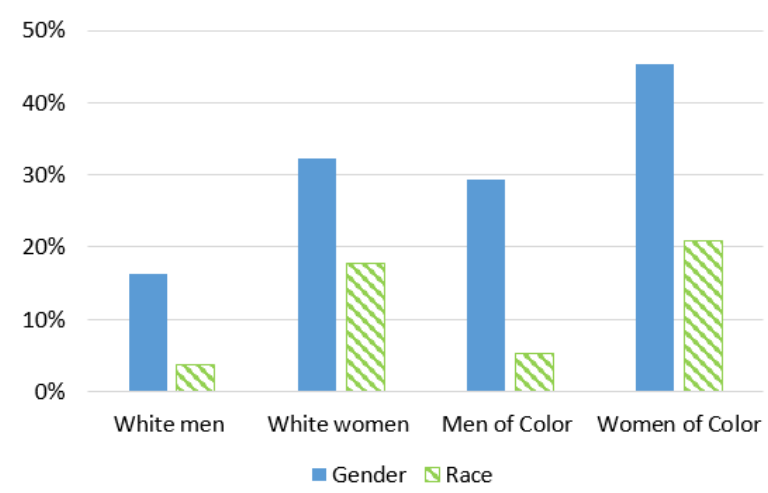

FIG 3. Percent of respondents attributing gender and race differences in STEM to societal beliefs about women and people of color.

about race. Figure 3 presents the reporting of societal beliefs impacting women and students of color by demographic group. Notably, men of all races discussed societal assumptions about race at similar rates.

Societal beliefs mentioned in the interviews ranged from stereotypes to explicit discrimination. For example, one black female major recounted: "I've had some professors who make it plain and clear that, no, because you're black you're not focused and I'm not really trying to, you know, give you the time of day." Notably, women were the most likely to mention societal beliefs based on both gender and race.

Some Races Lack Social and Cultural Capital. One unique code emerged for race differences in STEM: a lack of social and cultural capital. This includes access to educational tools, K-12 educational background, economic resources, and STEM-specific social support. Mentions of these factors appeared in direct response to the question posed about race differences. For example, one black female leaver stated: "[For] African Americans... there may be ones that were not privileged to go to such a nice high school. There may be a lack of exposure [to STEM]." No parallel reference to social/cultural capital was mentioned in regards to gender differences. Notably, no white men made a connection between race and social/cultural capital.

\section{Women and Students of Color Benefit from Their Underrepresentation}

Though not extremely common, several students mentioned benefits that women $(n=7)$ and students of color $(n=10)$ gain due to their underrepresentation. Advantages typically were related to job and scholarship opportunities that ostensibly are largely unavailable to students from majority groups, such as white and male students.

Some mentioned that professors are easier on women and students of color or that underrepresented students receive extra encouragement to pursue STEM fields. Responses coded in this way are not included in the analysis provided in Figure 1.

\section{CONCLUSION}

Responses were able to be categorized in three ways: (1) doesn't notice differences, (2) notes differences but only attributes them to ascribed characteristics of people, and (3) notes sexism/racism impacts. Ascribed characteristics reported shows that some students attribute differences in the experiences of women and URMs to something that is innate in those students. However, more students voiced explanations that are results of racism and sexism, such as stereotypes, discrimination, and underrepresentation.

We found white men were the least likely to recognize differences in the experiences of women and people of color in STEM. In contrast, women of color were the most likely to recognize those differences. Of notable importance, students of all demographics appeared more aware of gender differences than they were of race differences.

White men make up the majority in STEM, yet they are typically left out of research about and efforts to address inequity in STEM. This study suggests the majority population is largely in denial about the impacts of racism and sexism in STEM. Currently, most efforts to address inequity in STEM focus on helping women and people of color. We propose that efforts to address sexism and racism in STEM will be more successful if males and white people are viewed as being as integral to the solution as those from underrepresented groups.

\section{ACKNOWLEDGEMENTS}

We are grateful to the NSF for funding this project. NSF-STEP, DUE-0969286.

\section{REFERENCES}

1. National Science Foundation. 2017, Arlington, VA: Special Report NSF 17-310.

2. Strayhorn, T.L. 2012: Routledge.

3. Steele, C.M. et al. 2002. 34: p. 379-440.

4. Grossman, J.M. et al. 2014. 49(6): p. 698-727.

5. Walton, G.M. et al. Science, 2011. 331(6023).

6. Riffle, R., et al. Journal of Women and Minorities in Science and Engineering, 2013. 19(3).

7. Dryburgh, H. 1999. 13(5): p. 664-682.

8. Dancy, M. et al. 2016 PERC Proceedings.

9. Pew Research Survey of Adults, 2013.

10. Pew Research Survey of Adults, 2016.

11. Norton, M.I. et al. Perspectives on Psychological Science, 2011. 6(3): p. 215-218. 